\title{
EXAMINING PROBLEMATIC GAMBLING AND MENTAL HEALTH IN LGBTI COMMUNITIES: A PRELIMINARY STUDY
}

\author{
Philip Birch, \\ University of Western Sydney, Australia
}

Jane L. Ireland*, Ashworth Research Centre, Mersey Care NHS Trust and University of Central Lancashire, $U K$

Clare Strickland, Ashworth Research Centre, Mersey Care NHS Trust and University of Central Lancashire, $U K$ Johann Kolstee Senior Research Officer, ACON, Australia

*Corresponding author Professor Jane L. Ireland, JLIreland1@uclan.ac.uk; Ashworth Research Centre, Mersey Care NHS Trust and University of Central Lancashire, UK

\begin{abstract}
This preliminary study explored gambling in the lesbian, gay, bisexual, transsexual and intersex (LGBTI) communities, recognising this as an under researched area. Several factors were considered alongside gambling, namely mental health, substance use, alcohol use and self-control. Participants were recruited to take part in an online survey, with a final sample of 69 obtained. Findings revealed that gambling activities such as pub slot machines/games (58\%) were the most common form of gambling, followed by scratch cards $(43.5 \%)$. The most common motive for engaging in gambling was for 'fun', followed by 'because you like the feeling'. Twenty per cent of participants appeared to meet the criteria for gambling disorder, as defined by the DSM-V. Mental health variables, namely depression and anxiety, did not distinguish between problematic and non-problematic gambling whereas alcohol, drug use and self-control did; higher levels of alcohol and higher levels of drug use were present in the problematic gambling group which appeared to confirm gambling as a difficulty best placed within the addiction spectrum. Self-control was also lower in the problematic gambling group and represented a significant predictor of problematic gambling.
\end{abstract}

Keywords: Gambling disorder; LGBTI; Substance use; Gambling and mental health 


\section{INTRODUCTION}

Gambling can range from a recreational activity to one that is problematic in nature. The latter is characterised by chasing losses, lying to others about involvement in gambling, using it to cope and becoming preoccupied with gambling. It can lead to a range of negative impacts on cognitive and social functioning (APA, 2012). Gambling behaviour has recently been reclassified from an impulse control disorder to a disorder of addiction (DSM-V, APA 2012). This reclassification has been sought due to problematic gambling having several similarities to substance use disorders, including sharing patterns of development and maintenance (Grant, 2008). As such, reclassification in the DSM has highlighted the importance for (further) research to explore gambling behaviour with a specific focus on mental health and substance use disorders, as well as recognising the less problematic nature of gambling so the full spectrum of challenges can be ascertained. Although there is a growing body of research into gambling worldwide, limited attention has been paid to incorporating the lesbian, gay, bisexual, transsexual and intersex (LGBTI) communities into this research base.

Within the wider gambling literature research base there are two approaches adopted when exploring the association between gambling and mental health. One argues that gambling assists with coping and the management of mental health symptoms (Jacobs 1986; Blaszczynski \& McConaghy 1989), whereas the other argues that gambling can induce mental health issues such as depression or anxiety as a result of financial crises or other problems that can develop from engaging in gambling (Blaszczynski \& Nower, 2002). The association between these variables, and any likely protective factors such as selfcontrol (Clarke, 2004), have not received attention in LGBTI communities. This is not unique to the gambling literature, with research into addiction more broadly (e.g. alcohol, drugs) in LGBTI communities equally limited. Of the limited research conducted, it has been shown that LGBTI communities are more likely to misuse substances in comparison to non-LGBTI communities (Liao, 2015). A greater number of mental health difficulties have also been reported in LGBTI communities (Grant \& Potenza, 2006).

Consequently the current study represents a preliminary study that aims to explore gambling in LGBTI communities and examine the association between this, substance use and mental health. It aims to also examine if self-control presents as a factor impacting on any association. The research is novel and does not have existing research to draw on comprehensively. Thus, predictions are limited to broad indications of what could be expected, as follows: 1.) That problematic gambling will be associated with difficulties in mental health such as depression and anxiety; 2.) That selfcontrol will be associated with gambling, with decreased self-control predicting increased problematic gambling; 3.) That gambling and substance use will be positively associated.

\section{METHOD}

Participants: The sample was recruited by a recruitment campaign administered by ACON (ACON is the New South Wales LGBTI Health 
Organisation based in Sydney, Australia) using an on-line survey. The study sample comprised 109 returned measures of which $68.8 \%(\mathrm{n}=75)$ had completed the questionnaires. Five participants $(6.7 \%)$ were excluded from the data because they only completed the demographic questions. The final sample $(\mathrm{n}=69)$ included 44 $(63.8 \%)$ men, 21 (30.4\%) women, two $(2.9 \%)$ transgender and two (2.9\%) referring to themselves as unclear on their gender. The sample was aged between 18 and $72(M=36.5$, S.D $=13.5)$.

Measures: In addition to a demographic questionnaire, the following self-report measures were used. Gambling Motives Questionnaire (GMQ: Stewart \& Zack, 2008) consisting of fifteen items measuring the motivations for engaging in gambling; Gambling Disorder measure (GAM - DS), based on the DSM-V diagnostic criteria for problematic gambling in which participants were asked to describe their gambling and the extent to which it impacted on their lives across eight items; Hospital Anxiety and Depression Scale (HAD: Zigmond \& Snaith, 1983), which asked participants' to self-report how they had been feeling during the last couple of weeks, using 14 items; Alcohol Use Disorder Identification Test (AUDIT: Babor et al, 2001), which measured alcohol consumption in the past twelve months using ten items; Drug Abuse Screening Test - 10 (DAST - 10:
Skinner, 1982) measuring drug use in the last twelve months (excluding alcohol and tobacco) using ten items; and The Brief Self Control Scale (BSCS: Tangney, Baumeister \& Boone, 2004), a 13 item measure exploring participant self-control levels.

Procedure: An online survey link was provided to participants. Participants were informed of the requirements of the study, the benefits and the potential risks through the information sheet provided online, prior to participants engaging.

\section{RESULTS}

The results commence with an outline of the gambling behaviours reported prior to consideration of the association between gambling, mental health and substance use. The motivations for gambling are then outlined.

Nature of gambling and association with mental health, substance use and selfcontrol

Across the sample, 33.8 per cent reported being a current gambler (daily/weekly gambling), 58.8 per cent an occasional gambler (once a month or less) and 7.4 per cent a former gambler. The types of gambling engaged in are presented in Table 1.

Table 1. Forms of gambling (in rank order)

\begin{tabular}{ll}
\hline Gambling Activities (in order of frequency) & $\mathrm{n}(\%)$ Participants \\
\hline Pub Slot Machines/ games & $40(58)$ \\
Scratch Cards & $30(43.5)$ \\
Poker Games & $20(29)$ \\
Horse Racing & $20(29)$ \\
Bingo & $12(17.4)$ \\
Roulette & $9(13)$
\end{tabular}


Dog Racing

Other sport betting: AFL, football, World Cup AFL

Other - Card Tables

Other - Casino Tables

Other - Online Poker

Other - Private Gambling

Other - Raffle Tickets

Participants were categorised as problematic gamblers if they met four or more of the diagnostic criteria on the DSM-V measure. This resulted in 14 participants (20.2 per cent) being classified as problematic gamblers. Out of the 14,11 were men and three were women. The severity of problematic gambling was then grouped into mild, moderate or severe depending on number of criterion met, resulting in 43 per cent $(8.7$ per cent of the entire sample) being classified as mild, 36 per cent (7.2 per cent entire sample) as moderate and 21 per cent $(4.3$ per cent entire sample) as severe. The presentation of problematic and non-problematic gamblers on the depression, anxiety, substance use and self-control measures are presented in Table 2 .

Table 2. Means on mental health, substance use and self-control measure across the sample

\begin{tabular}{|c|c|c|c|}
\hline & Mean (S.D) & $\begin{array}{l}\text { Non - } \\
\text { problematic } \\
\text { gamblers } \\
\text { Mean (SD) }\end{array}$ & $\begin{array}{l}\text { Problematic } \\
\text { gamblers } \\
\text { Mean (SD) }\end{array}$ \\
\hline Depression (HAD) $\left(n=68,1 \mathrm{msg}^{*}\right)$ & $4.62(3.9)$ & $4.20(3.8)$ & $6.38(4.2)$ \\
\hline Anxiety (HAD) $(\mathrm{n}=68,1 \mathrm{msg} *)$ & $7.76(4.5)$ & $7.29(4.3)$ & $9.77(4.9)$ \\
\hline Drug use (DAST) $\left(\mathrm{n}=65,4 \mathrm{msg}^{*}\right)$ & $1.83(2.1)$ & $1.57(1.7)$ & $2.92(2.9)$ \\
\hline Alcohol use (AUDIT) $(\mathrm{n}=62,7 \mathrm{msg} *)$ & $9.87(7.1)$ & $8.86(5.8)$ & $13.69(10.3)$ \\
\hline Self - Control $\left(n=63,6 \mathrm{msg}^{*}\right)$ & $42.8(9.9)$ & $44.7(9.3)$ & $34.7(9.1)$ \\
\hline
\end{tabular}

$*=$ missing

ANOVA analyses were conducted to explore differences between problematic and non-problematic gamblers. There was no significant difference with regards to depression $(F(1,66)=3.40 \mathrm{~ns})$ or anxiety $(F(1,66)=3.35 \mathrm{~ns})$. There was a significant difference in relation to alcohol use $(F(1,60)=5.00$, MSE $=240.20, p<$ $.03)$, with problematic gamblers reporting greater levels than non-problematic gamblers. This was replicated for drug use $(F(1,64)=4.73$, MSE $=19.23, p<.03)$. Finally, regarding levels of self-control, this was significantly different between problematic and non-problematic gamblers $(F(1,61)=11.46, \mathrm{MSE}=979.1, p<.001)$, with lower levels of self-control in the problematic gambling group.

A multiple regression was then conducted to examine whether these variables (anxiety, depression, alcohol use, drug use and self-control) were significant predictors of problematic gambling. The overall model was significant $(F(5,53)=$ 3.61, $\mathrm{MSE}=15.78, p<. .01)$. Of the entered variables only (lower) self-control 
was a significant predictor of problematic levels of gambling $(\beta=-.38, t=-2.20, p<$ $.03)$.
Motivations for gambling

The motivations for gambling behaviour are presented in Table 3 .

Table 3. Gambling motives overall and between problematic and non-problematic gamblers

\begin{tabular}{llcc}
\hline \multicolumn{1}{c}{$\begin{array}{c}\text { GMQ } \\
\text { (15 items: presented in overall rank } \\
\text { order) }\end{array}$} & $\begin{array}{c}\text { Overall } \\
\text { (Mean/SD) }\end{array}$ & $\begin{array}{c}\text { Non- } \\
\text { problematic } \\
\text { gamblers } \\
\text { (Mean/SD) }\end{array}$ & $\begin{array}{c}\text { Problematic } \\
\text { gamblers } \\
\text { (Mean/SD) }\end{array}$ \\
\hline $\begin{array}{l}\text { Because it's fun } \\
\text { Because you like the feeling }\end{array}$ & $2.29(1.02)$ & $2.15(0.93)$ & $2.86(1.17)$ \\
Because it's exciting & $2.16(1.01)$ & $1.93(0.92)$ & $3.07(0.83)$ \\
Because it is something I do on special & $2.14(0.96)$ & $1.95(0.87)$ & $2.93(0.92)$ \\
occasions & $1.81(0.81)$ & $1.73(0.76)$ & $2.14(0.95)$ \\
To relax & $1.75(0.95)$ & $1.48(0.72)$ & $2.79(1.05)$ \\
Because it makes you feel good & $1.75(0.85)$ & $1.55(0.69)$ & $2.57(0.94)$ \\
To forget your worries & $1.71(0.97)$ & $1.38(0.71)$ & $3.00(0.78)$ \\
To get a 'high' feeling & $1.70(0.93)$ & $1.44(0.71)$ & $2.71(0.99)$ \\
To be sociable & $1.70(0.77)$ & $1.60(0.71)$ & $2.07(0.92)$ \\
As a way to celebrate & $1.65(0.73)$ & $1.55(0.72)$ & $2.08(0.64)$ \\
To cheer you up when you're in a bad mood & $1.62(0.94)$ & $1.29(0.60)$ & $2.93(0.92)$ \\
Because it helps when you are feeling & $1.61(0.90)$ & $1.36(0.73)$ & $2.57(0.85)$ \\
nervous or depressed & & & \\
Because it makes a social gathering more & $1.57(0.76)$ & $1.45(0.66)$ & $2.00(0.96)$ \\
enjoyable & & & \\
Because it's what most of your friends do & $1.54(0.70)$ & $1.46(0.67)$ & $1.86(0.77)$ \\
when you get together & & & $2.00(0.78)$ \\
Because you feel more self - confident or & $1.32(0.59)$ & $1.13(0.35)$ & \\
sure of yourself & & & \\
\hline
\end{tabular}

Problematic gamblers were significantly more likely than nonproblematic gamblers to report the following (using ANOVA analysis); Because it's fun $(p<.02)$; Because you like the feeling $(p<.001)$; Because it's exciting $(p<.001)$; To relax $(p<.001)$; Because it makes you feel good $(p<.001)$; To forget your worries $(p<.001)$; To get a 'high' feeling $(p<.001)$; To be sociable ( $p$ $<.04)$; As a way to celebrate $(p<.05)$; To cheer you up when you're in a bad mood $(p<.001)$; Because it helps when you are feeling nervous or depressed $(p<.001)$; Because it makes a social gathering more enjoyable $(p<.02)$; Because you feel more self-confident or sure of yourself $(p<$ $.001)$.

A principle component analysis (PCA) method was adopted to explore the factors underlying the gambling motives reported. This produced three factors using items with factor loadings of at least .40 , with only one item failing to load. 
The three factors were Factor 1 (48\% variance): 'Mood enhancement' comprising four items focusing on gambling as a means of positively improving or elevating mood; Factor 2 (14.7\% variance): 'Socialising/celebration' comprising five items centred on gambling as a means of social reward and engagement; Factor 3 (9.5\% variance): 'Coping' comprising five items where gambling was used as a mean of managing negative cognition and physiological arousal. These factors were then calculated into factor scores (i.e. items loading above .50 in each factor), with the results presented in Table 4.

Table 4. Gambling motivation factors across problematic and non-problematic gamblers.

\begin{tabular}{lll}
\hline Gambling Motivations & $\begin{array}{l}\text { Non-problematic } \\
\text { gamblers }\end{array}$ & $\begin{array}{l}\text { Problematic } \\
\text { gamblers }\end{array}$ \\
& Mean (SD) & Mean (SD) \\
\hline Mood enhancement & $8.94(3.47)$ & $14(3.67)$ \\
Socialising/ celebration & $7.76(2.36)$ & $10(3.29)$ \\
Coping & $6.35(2.20)$ & $13.2(2.71)$ \\
\hline
\end{tabular}

A MANOVA noted a significant difference between problematic and nonproblematic gambling on each of the motivations $(F(1,64)=30.4, p<.001)$. The subsequent univariate analyses found problematic gamblers scored higher on all factors than non-problematic gamblers (i.e. mood enhancement; $F(1,64)=21.5, p<$ .001 ; socialising/celebration, $F(1,64)=7.8$, $p<.01$; coping, $F(1,64)=92.1, p<.001)$.

\section{DISCUSSION}

This preliminary study demonstrates the importance of examining problematic gambling behaviour in LGBTI communities. The finding that a fifth of the sample met the DSM-V criteria for problematic gambling is significant and warrants further examination. The most common form of gambling reported represented pub slot machines/games (58 per cent), followed by scratch cards (43 per cent), poker games (29 per cent) and horse racing (29 per cent). Thus, there was considerable diversity in the nature of gambling.

Of specific interest were the variables associating with problematic gambling, particularly when accounting for the size of the sample acquired and therefore the strength of these findings. Consistent with the prediction made, problematic gamblers were reporting higher levels of drug and alcohol use compared to those classified as nonproblematic gamblers. Inconsistent with the prediction, however, was the finding that mental health variables (i.e. depression and anxiety) were not associating. This would suggest that the reclassification of gambling in DSM-V as more closely aligned to an addictive disorder is supported. The absence of a relationship between gambling and mental health variables (depression and anxiety) was unexpected, particularly when accounting for research suggesting that gambling is either a means of assisting with mental health symptoms (Jacobs 1986; 
Blaszczynski \& McConaghy 1989) or a factor important in inducing mental health challenges (Blaszczynski \& Nower, 2002). However, the current research did not examine the other factors that may associate with gambling (e.g. financial pressures) which could then produce difficulties with mental health (Blaszczynski \& Nower, 2002). It could be speculated, for example, that the current sample was high-functioning in that their gambling and associated use of substances were not causing problems that extended into mental health difficulties more broadly.

Indeed, it was self-control levels that were most important in predicting problematic gambling. This was the only significant predictor within the regression model and it supported the hypothesis that lower self-control would predict higher levels of problematic gambling. The importance of (lower) self-control is consistent with previous research (Clarke, 2004), including that extending to substance use, thereby suggesting a further association with substance use challenges replicated in the current sample.

Gambling motivations were also found to be multi-faceted, broadly comprising of gambling to improve or elevate mood; to socialise; or to cope with negative affect and cognition. Problematic gamblers consistently reported higher levels of all motivations than nonproblematic gamblers. This indicates an absence of discriminating differences across these motivations and that some motivations are likely to be positively reinforcing (i.e. to improve/elevate mood, to socialise) with only one set of motivations likely to be negatively reinforcing (i.e. removal of negative affect/cognition). Future research examining the influence of these motivations across time would be of value since it could be speculated that the positively reinforcing motivations are serving to protect against poorer mental health.

The current research is not without its limitations; the sample is small and likely self-selecting, with no objective measures taken of the variables of interest. Nevertheless, as a preliminary study into gambling it highlights the importance of promoting further research into this area, across a range of communities, and appears to add to the importance of considering gambling as aligned more closely to addictive disorders than broader mental health challenges. 


\section{REFERENCES}

American Psychiatric Association (APA) (2012). Diagnostic and statistical manual of mental disorders - Text Revision. (5th ed.). Washington, DC: American Psychiatric Association.

Babor, T., Higgins-Biddle, J.C, Saunders, J. B, Monteiro, M. G. (2001). AUDIT - The Alcohol Use Disorders Identification Test: Guidelines for Use in Primary Health Care (2nd edn). Geneva: World Health Organisation.

Blaszczynski, A., McConaghy, N. (1989) Anxiety and/or depression in the pathogenesis of addictive Gambling. International Journal of the Addictions, 24, 337-350.

Blaszczynski, A., Nower, L. (2002). A pathways model of problem and pathological gambling. Addiction, 97, 487499.

Blume, S. (1987). Compulsive gambling and the medical model. Journal of Gambling Behaviour, 3, 237-247

Clarke, D. (2004). Impulsiveness, locus of control, motivation and problem gambling. Journal of Gambling Studies, 20, 319-345.

Grant, J. E., Potenza, M. N. (2006). Sexual orientation of men with pathological gambling: Prevalence and psychiatric comorbidity in treatmentseeking sample. Comprehensive Psychiatry, 47, 515-8.

Grant, J. (2008). Gambling problems in Gay/ Bisexual men. NALGAP the association of lesbian, gay, bisexual, transgender addiction professionals and their allies, XX1 (1), $1-8$.

Liao, M. (2015) Problem Gambling and the lesbian, gay, bisexual and transgender community, NAPAFASA Problem Gambling Prevention Technical Assistance and Training Project Funded by the State Department of Alcohol and Drug Programs, Office of Problem Gambling. Retrieved March 2015 from http://www.nsgamingfoundation.org/uploa ds/Research/Problem\%20Gambling\%20an d\%20the\%20LGBT.pdf

Stewart, S. H., Zack, M. (2008). Development and psychometric evaluation of a three-dimensional Gambling Motives Questionnaire. Addiction, 103, 1110 1117.

Skinner, H. A. (1982). The Drug Abuse Screening Test. Addictive Behaviour, 7, 363-371.

Tangney, J P., Baumeister, R. F., Boone, A. L. (2004). High self-control predicts good adjustment, less pathology, better grades, and interpersonal success. Journal of Personality, 72, 271 - 324.

Zigmond, A. S., Snaith, R. P (1983). The hospital anxiety and depression scale. Acta Psychiatr Scand, 67, 361-370. 